\title{
Two-photon laser scanning fluorescence microscopy using photonic crystal fibre
}

\author{
Gail McConnell $^{1 *}$ and Erling Riis ${ }^{2}$ \\ ${ }^{1}$ Centre for Biophotonics, SIBS, 27 Taylor Street, University of Strathclyde, Glasgow G4 ONR, \\ Scotland \\ ${ }^{2}$ Department of Physics, John Anderson Building, University of Strathclyde, Glasgow G4 ONG, \\ Scotland \\ Tel: $+44(141) 5484738$ \\ Fax: $+44(141) 5484887$ \\ Email: g.mcconnell@strath.ac.uk
}

Keywords: Multi-photon, microscopy, fluorescence, ultrafast, nonlinear.

\begin{abstract}
We report the application of a simple yet powerful modular pulse compression system, based on photonic crystal fibres which improves upon incumbent twophoton laser scanning fluorescence microscopy techniques. This system provided more than a 7-fold increase in fluorescence yield when compared with a commercial two-photon microscopy system. From this, we infer pulses of infrared radiaton of less than $35 f_{s}$ duration reaching the sample.
\end{abstract}




\section{Introduction}

Multi-photon laser scanning microscopy (MPLSM) is a well-established tool for deep and live imaging of biological tissue ${ }^{1}$. Two types of laser source suited to two-photon excitation exist, namely continuous wave (cw) and ultra-short pulsed (USP) lasers, the latter delivering pulses typically of sub-picosecond duration every few nanoseconds. The resulting peak power from USP lasers can easily reach the kilowatt level, whilst retaining a modest average power level. The very high peak powers generated by USP laser sources are ideal for multi-photon applications when focussed using an objective lens. The primary advantage of employing an USP laser for two-photon laser scanning microscopy (TPLSM) over a cw alternative is an increase in sample viability thanks to the small duty cycle. This permits sample and fluorophore recovery on the nanosecond scale. An additional advantage of employing an USP source for TPLSM is the conservative average power delivered. This reduces detrimental heating effects through linear absorption that are observed when applying a cw laser source ${ }^{2}$.

The most popular choice of USP laser gain medium for TPLSM is currently Ti:Sapphire. Commercial lasers based on this material can support wavelengths from 720-930 nm with a single mirror set, and may deliver pulses as short as $140 \mathrm{fs}$ duration ${ }^{3,4}$. The laser source is typically used in conjunction with a computer-controlled scan-head that manipulates the beam profile of the entering laser, and a microscope with a high ( $>10 x)$ magnification objective lens to focus the radiation onto the prepared biological sample for investigation and subsequent image capture. Therefore, before the laser pulse reaches the sample it is relayed by and propagated through a number of optical elements, including steering mirrors, beam-shaping optics and filters. Unfortunately, each of these elements will stretch the pulse through dispersion. As a consequence, the duration of the pulse reaching the sample is commonly much longer than that emitted by the USP laser. Efforts have been made to pre-compensate for dispersion by including prisms in a home-built laser resonator, delivering pulses of 15.5 fs duration ${ }^{5}$, but this approach is inflexible when used with alternative commercial resonators.

To solve this problem, we have developed an effective pulse compression technique based on photonic crystal fibre (PCF). Previous studies by Ouzounov et. al. have demonstrated that 
ultrashort pulses can propagate through PCF but in their investigation the emitted pulses are stretched from $100 \mathrm{fs}$ to $140 \mathrm{fs}^{6}$. We report on a system which compresses the pulse duration and, using a conventional Ti:Sapphire laser platform and standard MPLSM equipment, returns more than a seven-fold increase of the fluorescence signal (i.e. fluorescence yield) from prepared biological samples at standard excitation wavelengths. This is of particular benefit for fluorophores that are only weakly excited by standard commercially available laser units. Our modular system is an ideal accompaniment to most commercial laser sources, and comprises very basic and fixed opto-mechanical components to facilitate ease of use.

The development of $\mathrm{PCF}^{7}$ has brought renewed interest to the field of non-linear fibre optics. PCFs are micro-structured fibres, where the light is guided by a number of periodically arranged air holes that extend along the length of the fibre. These holes create a photonic bandgap in the transverse dimension resulting, for instance, in fibres that are single-mode throughout the visible range $^{8}$. Alternatively, this superior guiding property can be viewed as resulting from the large refractive index step between the fused silica core and the surrounding partly air filled cladding. This large refractive index step also allows for a reduction in the core diameter down to a few microns, leading to a significant increase in the propagating peak intensity. This is of obvious benefit in the study of non-linear effects, but just as significant is the fact that the exact nature of the micro-structure determines the group velocity dispersion (GVD) of the fibre. Typically, the zero-point, $\lambda_{0}$, for the GVD in a $1-2 \mu \mathrm{m}$ diameter PCF is shifted from the bulk silica value of around $1300 \mathrm{~nm}$ down to $600-800 \mathrm{~nm}^{8}$. This means that it is now possible to have a fibre with anomalous dispersion at a convenient wavelength, such as that of the Ti:Sapphire laser. The most spectacular manifestation of this high non-linearity in an anomalously dispersive regime is the generation of a white-light supercontinuum which in part is due to soliton formation ${ }^{9,10}$.

The situation is far simpler for wavelengths below the zero-point for the group velocity dispersion. The normal dispersion prevents soliton formation, leaving self-phase modulation as the main non-linear effect. In this paper we investigate a dispersion pre-compensation scheme based on spectral broadening in a normally dispersive PCF and subsequent dispersion compensation in a grating pair. We show how the unique properties of the fibre in terms of high non-linearity and low GVD combine to give ideal conditions for ultra-short pulse compression. The 250 fs pulses from a commercial Ti:Sapphire laser are compressed to $<35$ fs at the 
microscope stage. This results in more than a 7 -fold increase in fluorescence detected from the biological sample. This method has significant potential for improvements through a dedicated PCF design, providing a simple and effective route to obtaining a pulse of sub-10 fs duration at the sample, where laser operation is significantly more challenging.

\section{Experimental}

A schematic of the PCF-enabled laser experimental set-up is shown in Fig. 1A. A commercial Ti:Sapphire laser (Coherent, Mira 900-F) emitted a $76 \mathrm{MHz}$ train of pulses in the region $800-$ $860 \mathrm{~nm}$, with the pulse width measured to be approximately 250 fs with an interferometric autocorrelation trace consistent with a $\operatorname{sech}^{2}$ pulse shape. The same laser was used both directly and as the pump for the PCF-enabled system in order to directly compare performance when employed as an excitation source for TPLSM.

In the PCF-enabled system, this light was propagated through a Faraday isolator to reduce feedback from the PCF facets, which largely limited the wavelength range of operation. The output was focused into an $86 \mathrm{~mm}$ length of PCF using a spherical anti-reflection coated $f=+4.5$ $\mathrm{mm}$ lens. The output was collimated and passed through a dispersion compensating stage, consisting of two parallel 600 lines/mm gratings separated by up to $16 \mathrm{~mm}$. This grating pair introduced a negative dispersion in the beam of $b_{0} \cdot 0.463 \mathrm{ps}^{2} / \mathrm{m}$, where $b_{0}$ was the separation between the gratings ${ }^{11}$. The light transmitted by the PCF was then passed into a scan-head (Bio-Rad, MRC1024ES) coupled to an inverted microscope (Nikon, TE300), as shown in figure 1B. Alternatively, the conventional Ti:Sapphire excitation light was similarly passed into the scan-head and microscope system. The light entering the scan-head was reflected and manipulated by scanning mirrors towards the microscope. A 20x/0.75 numerical aperture air microscope objective lens (Nikon, Plan Fluor) was used to focus the radiation onto the fluorescently stained sample. Fluorescence resulting from two-photon excitation, using either the PCF-augmented laser or conventional Ti:Sapphire source, was collected by the same objective lens and propagated through an optical bandpass filter to reject reflected light from the exciting source. The fluorescence was then relayed to a sensitive photomultiplier tube. This 
signal was used, along with image capture software, to visualise fluorescently stained regions of the sample.

The PCF was manufactured by Crystal Fibre A/S. It was made of pure silica and had a hexagonal structure of air holes to guide the light in a $2.6 \mu \mathrm{m}$ core. The distance between adjacent air holes was $1.8 \pm 0.2 \mu \mathrm{m}$ and the hole size to separation ratio was approximately 0.35 . This structure gave rise to a high non-linearity and a zero dispersion point around $900 \mathrm{~nm}$. The fibre therefore had a low and positive dispersion throughout the normal Ti:Sapphire operating range $(720-890 \mathrm{~nm})$ and is therefore suitable for pulse compression across this range. For a longer operational input wavelength range, e.g. 900-1000nm, a slightly different fibre with a longer-wavelength zero-dispersion point would be required. The dispersion in the fibre used was estimated to be $50 \mathrm{ps} / \mathrm{nm} \cdot \mathrm{km}$ at $800 \mathrm{~nm}$.

Prior to the application of the PCF-assisted system for TPLSM, we quantified the operational properties of the fibre. A full description of the analysis is described elsewhere ${ }^{12}$, therefore here we present only the key outcomes. Figure $2 \mathrm{~A}$ shows a typical transmission spectrum of the fibre output with $188 \mathrm{~mW}$ of light transmitted through $86 \mathrm{~mm}$ of fibre (representing a transmission of $61 \%$ ). This corresponded to a pulse energy of $\sim 2.35 \mathrm{~nJ}$ at a laser wavelength of $\lambda=830 \mathrm{~nm}$. The spectrum shows the multi-peak structure characteristic of self-phase modulation with a broadening to $40 \mathrm{THz}$. Additionally, the spectral bandwidth was observed to increase linearly with the power transmitted through the fibre. The combined effect of the non-linear self-phase modulation and the positive dispersion in the PCF was an almost linear frequency variation across the pulse (chirp). It was this linear variation that enabled the use of the anomalous dispersion of a grating pair to compress the pulse.

In order to experimentally quantify the pulse shape from the fibre and grating pair we recorded the interferometric autocorrelation signal for the pulse, using a home-made collinear scanning autocorrelator based on two-photon absorption in a GaAsP photodiode ${ }^{13}$. Figure $2 \mathrm{~B}$ shows an example of the recorded trace for $175 \mathrm{~mW}$ transmitted through the fibre at a wavelength of 830 $\mathrm{nm}$. The initial grating separation was chosen to maximise the peak intensity or, equivalently, the autocorrelation signal. This was where we would expect the shortest pulses and hence the maximum two-photon response from the photodiode. The signal displayed the familiar 
symmetric shape with a peak to background ratio of $8: 1$. The notable difference compared with the familiar $\operatorname{sech}^{2}$ autocorrelation signal was the prominent structure in the wings due to side lobes on the pulse. The main part of the pulse is a narrow central feature containing $75 \%$ of the energy, while the remaining energy is distributed symmetrically in two side lobes immediately adjacent to the central peak.

We calculated the ratio of the full-width at half-maximum of a typical pulse and the number of interference fringes across the autocorrelation trace to be a constant $1.84 \mathrm{fs} /$ fringe or slightly larger than the ratio of $1.44 \mathrm{fs} /$ fringe for a $\mathrm{sech}^{2}$ pulse. The 13.5 fringes observed in the experimental data shown in figure 4 therefore correspond to a pulse width of $24.8 \mathrm{fs}$ or an order of magnitude compression of the original laser pulse.

To demonstrate the benefits of the dispersion-compensated system, we performed TPLSM using both the PCF-enabled pre-compensation system and the commercial Ti:Sapphire laser unit. The first sample imaged was guinea pig small intestine muscularis externa labelled with an anti-smooth muscle myosin primary antibody and Alexa 594-conjugated goat anti-mouse secondary antibody. To facilitate a complete comparison when used for direct two-photon excitation, the average power of the commercial source was set to $24 \mathrm{~mW}$ to exactly match that from the PCF-enabled system. Additionally, the output from the PCF-enabled system was manipulated to replicate the spatial characteristics of the commercial system to ensure identical filling of the objective lens. The imaging and capture system was identical in both configurations.

\section{Results}

Figure $3 \mathrm{~A}(\mathrm{a}-\mathrm{O})$ shows a series of images taken with the PCF-enabled system when $24 \mathrm{~mW}$ of average power was delivered into the scanhead, as configured in figure 2. The images were taken while changing the grating spacing from $9 \mathrm{~mm}$ to $16 \mathrm{~mm}$ and hence altering the effective pulse duration at the sample. For comparison, figure $3 \mathrm{~A}(\mathrm{p})$ shows the image obtained from applying the conventional Ti:Sapphire laser at the same average power. The images were taken at a capture rate of $\sim 1 \mathrm{~Hz}$, at $256 \times 256$ pixels box size. The repetition rate, average power and spatial beam properties of both the PCF-assisted and the conventional Ti:Sapphire laser 
systems were identical: the only difference was the pulse duration and hence the peak intensity of the radiation reaching the sample.

In comparison with the conventional excitation source, a more than seven-fold increase in fluorescence yield was consistently obtained under two-photon excitation of the sample with the PCF-enabled system. This is shown in figure 3B, where a 7.3-times increase in fluorescence signal intensity was measured using image analysis software (LaserPix, Bio-Rad) when switching from the conventional Ti:Sapphire laser source to the PCF-assisted laser for the sample described in figure $3 A(a-0)$. This data is taken from a sample of $n=10$ and is normalised with respect to the fluorescence yield from conventional Ti:Sapphire excitation. From this, we inferred that the pulse duration at the sample was more than a factor of seven times shorter using the PCF-enabled system. We therefore calculated a pulse duration of $<35 \mathrm{fs}$ arriving at the sample. This was more than an order of magnitude shorter than was previously measured using another commercial TPLSM system ${ }^{14}$. By attenuating the PCF-assisted source, a nearquadratic relationship (gradient of $1.8 \pm 0.1, n=20$ ) between the average fluorescence signal intensity and increasing average laser power was observed at a grating spacing of $12 \mathrm{~mm}$, as shown in figure $3 \mathrm{C}$. This confirmed the nonlinear nature of the excitation mechanism.

This grating separation was somewhat larger than the few $\mathrm{mm}$ found to be optimal for the direct pulse measurements ${ }^{12}$. We attribute this to the positive dispersion of the microscope system, which is effectively being pre-compensated by an increased negative dispersion from the grating pair. The fact that we do not observe a full 10 -fold increase in the signal that might be anticipated from the order of magnitude reduction in the pulse width most probably results from the increased higher order dispersion of the optical system, which is not compensated in the present implementation.

Similar experiments comparing the PCF-enhanced system with the normal Ti:Sapphire laser by imaging smooth muscle cells in intact, living rat pulmonary artery, labelled with the voltage sensitive dye di-8-ANEPPS were also performed. The images were taken at a capture rate of $\sim 1 \mathrm{~Hz}$, at $256 \times 256$ pixels box size, using a $60 \times / 1.4$ numerical aperture oil microscope objective lens (Nikon). The repetition rate, average power and spatial beam properties of both the PCFassisted and the conventional Ti:Sapphire laser systems were identical; again, the only difference was the pulse duration of the radiation reaching the sample. With an average power 
of $22 \mathrm{~mW}$ from both systems directed into the scanhead, Figure $4 \mathrm{~A}$ shows the images obtained with the PCF-enabled source (a) and the conventional Ti:Sapphire laser (b) at a grating spacing of $14 \mathrm{~mm}$. This $2 \mathrm{~mm}$ increase in grating spacing from the previous investigation of guinea pig small intestine muscularis externa compensated for the positive dispersion arising from the immersion oil and the alternative microscope objective lens used. Application of the PCFenabled laser source resulted in a 7.8-fold enhancement of fluorescence intensity when compared with direct excitation from the commercial Ti:Sapphire laser. This is shown in figure 4B. The TPLSM imaging process did not observably compromise either of the samples investigated, although previous reports indicate that the probability of cell destruction through TPLSM increases with a decrease in pulse duration ${ }^{15}$.

Over a typical imaging period of nine hours, no alignment of the PCF or associated beamsteering optics was required to maintain a $>7$-fold enhancement of fluorescence intensity compared with conventional excitation. Additionally, no short-term instability was observed during image capture at a rate of $1 \mathrm{~Hz}$.

These results demonstrate the considerable potential of the PCF-augmented system as a practical addition to markedly improve existing MPLSM systems for both fixed and live sample imaging. This is of particular importance in the instance of samples with weakly responsive fluorophores or those with only a small overlap of absorption wavelength with the emitting wavelength of the exciting USP laser source. Additionally, the increase in peak power due to the reduction in pulse duration indicates that the average power of the laser source can be decreased. This should improve sample integrity through reduced linear absorption of the excitation source.

\section{Discussion}

Although we have realized more than a seven-fold increase in fluorescence yield, it should be possible to increase this further by optimising the pulse compression conditions of the PCF, based on a fixed USP laser. The optimum condition for pulse compression based on standard, single-mode step-index fibres was determined theoretically by Tomlinson et al..$^{11}$ and applies 
equally well to the PCF system. The optimum compression factor $F_{\mathrm{c}}$ was found to have the following dependence on input parameters:

$$
F_{c} \propto T_{0} \sqrt{\frac{I_{0}}{\left|\beta_{2}\right|}}
$$

where $I_{0}$ was the peak intensity in the pulse, $T_{0}$ the input pulse duration, and $\beta_{2}$ the group velocity dispersion. This expression immediately shows the advantage of using a PCF. The small fibre cross-section compared with conventional optical fibre leads to a high peak intensity and the PFC provides the freedom to choose a suitably low group velocity dispersion for the particular wavelength region of interest. Applying relevant experimental fibre parameters to the detailed theory we found a maximum compression factor of 35 , which would be obtained at a fibre length of $40 \mathrm{~cm}^{12}$. We were forced to use an approximately five times shorter than optimal length of fibre, as the bandwidth of the pulse would otherwise have extended into the anomalously dispersive regime. The observed compression factor is therefore in reasonable qualitative agreement with this theory.

As mentioned above the high compression factor obtainable with a PCF is partly due to the relatively small group velocity dispersion. This, in turn, is at least partly due to the laser operating near the zero-point for the dispersion. In our experiments, this was too close for the required bandwidth for an optimally compressed pulse. However, the dispersive properties of PCFs can to some extent be tailored ${ }^{8,9}$ offering the enticing prospect of designing a fibre structure optimised for pulse compression. The fibre would have a small and highly non-linear core and a relatively small, positive, and as near as possible constant GVD over a bandwidth of a few hundred $\mathrm{nm}^{16}$. This would allow the same piece of fibre to be used over a wide wavelength range. We will experimentally investigate this optimisation process in due course. 


\section{Conclusion}

We have demonstrated the use of PCF in combination with a grating pair for pulse compression of $250 \mathrm{fs}$ Ti:Sapphire laser pulses to below $25 \mathrm{fs}$, and the subsequent improvement in TPLSM when applying conventional commercial systems. More than a seven-fold increase in fluorescence intensity yield was routinely measured using simple image analysis methods. From this we deduce a factor of seven reduction in the pulse duration reaching the sample to $<35$ fs. We have also investigated the ideal parameters for optimum pulse compression, and we anticipate that sub-10 fs pulses at the sample can be achieved using this technique. The experimental configuration was very basic, requiring comparable opto-mechanical precision with commercial imaging solutions, yet offered an unrivalled increase in fluorescence enhancement.

\section{Acknowledgements}

This work was carried out in collaboration with NKT Research and Innovation A/S and Crystal Fibre A/S, from whom the fibres were obtained. The authors acknowledge Ross Davidson, Karen McCloskey and Alison Gurney for supplying and preparing the samples used in this investigation. 


\section{References}

1. W. Denk, J.H. Strickler and W.W. Webb. 1990. Two-photon laser scanning fluorescence microscope. Science. 248: 73-76.

2. A. Diaspro, and M. Robello. 2000. Two-photon excitation of fluorescence for threedimensional optical imaging of biological structures. Journal of Photochemistry and Photobiology B: Biology. 55: 1-8.

3. Commercial product: Chameleon (Coherent Inc).

4. Commercial product: Mai-Tai (Spectra Physics).

5. M. Müller, J. Squier, R. Wolleschensky, U. Simon and G.J. Brakenhoff. 1998. Dispersion precompensation of 15 femtosecond optical pulses for high-numerical-aperture objectives. Journal of Microscopy 191: 141-150.

6. D.G. Ouzounov, K.D. Moll, M.A. Foster, W.R. Zipfel, W.W. Webb and A.L. Gaeta. Delivery of nanjoule femtosecond pulses through large-core microstructured fibers. Optics Letters 27: 1513-1515.

7. J.C. Knight, T.A. Birks, P. St. J. Russell and D.M. Atkin. 1996. All-silica single-mode optical fiber with photonic crystal cladding. Optics Letters 21: 1547-1549.

8. T.A. Birks, J.C. Knight and P. St. J. Russell. 1997. Endlessly single-mode photonic crystal fiber. Optics Letters. 22: 961-963.

9. J.K. Ranka, R.S. Windeler and A.J. Stentz. 2000. Visible continuum generation in air silica microstructure optical fibers with anomalous dispersion at 800nm. Optics Letters 25: 25-27.

10. A.V. Husakou, and J. Hermann. 2001. Supercontinuum generation of higher-order solitons by fission in photonic crystal fibers. Physical Review Letters. 87: 203901-203904.

11. W.J. Tomlinson, R.H. Stolen and C.V. Shank. 1984. Compression of optical pulses chirped by self-phase modulation in fibers. Journal of the Optics Society of America B 1: 139-149.

12. G. McConnell, and E. Riis. 2004. Ultra-short pulse compression using photonic crystal fibre. Accepted for publication in Applied Physics B.

13. J.K. Ranka, A.L. Gaeta, A. Baltuska, M.S. Pshenichnikov and D.A. Wiersma. 1997. Autocorrelation measurement of 6-fs pulses based on the two-photon-induced photocurrent in a GaAsP photodiode. Optics Letters 22: 1344-1346.

14. D. Wokosin, (private communication). 
15. K. König, T. W. Becker, P. Fischer, I. Riemann, and K.-J. Halbhuber. 1999. Pulse-length dependence of cellular response to intense near-infrared laser pulses in multiphoton microscopes. Optics Letters 24: 113-115.

16. A. Ferrando, E. Silvestra, J.J. Miret and P. Andrés. 2000. Nearly zero ultraflattened dispersion in photonic crystal fibers. Optics Letters 25: 790-792. 


\section{Figure legends}

Figure 1A. The output of a mode-locked Ti:Sapphire laser (Coherent Mira 900-F) is sent through a Faraday isolator (F.I.) and coupled into a section of photonic crystal fibre (PCF). The spectrally broadened output is compressed by the negative dispersion of a grating pair. The resultant radiation is then directed into a commercial scan-head (Bio-Rad MRC 1024ES).

Figure 1B. The light transmitted by either the PCF-enabled or the standard system was entered into a scan-head (Bio-Rad, MRC1024ES) coupled to an inverted microscope (Nikon, TE300). The light entering the scan-head was reflected and manipulated by scanning mirrors towards the microscope. A 20x/0.75 NA microscope objective lens (Nikon, Plan Fluor) was used to focus the radiation onto the fluorescently stained sample. Fluorescence resulting from two-photon excitation was collected by the same objective lens and relayed to a sensitive photomultiplier tube (PMT). This signal was used, along with image capture software, to visualise fluorescently stained regions of the sample in the conventional method.

Figure 2A. The spectrum of the light transmitted through $86 \mathrm{~mm}$ of fibre at an average transmitted power of $188 \mathrm{~mW}$. The spectrum shows a symmetric and approximately $38 \mathrm{THz}$ wide distribution (FWHM) with 9 individual maxima.

Figure 2B. Experimentally recorded autocorrelation signal for an optimally compressed pulse at $175 \mathrm{~mW}$ transmitted power. The $\sim 13.5$ fringes FWHM of this pulse correspond to a compressed pulse width of 25 fs. The dispersion required for optimum compression prior to entering the scan-head is is $1.22 \times 10^{-3} \mathrm{ps}^{2}$. 
Figure 3A. A series of TPLSM images of guinea pig small intestine muscularis externa labelled with anti-smooth muscle myosin an Alexa 594-conjugated antibody for smooth muscle myosin, using both the PCF-enabled system (a-o) and the commercial Ti:Sapphire laser unit (p). The grating spacing of the PCF-enabled system was increased from $9 \mathrm{~mm}$ (a) to $16 \mathrm{~mm}$ (o) in $0.5 \mathrm{~mm}$ increments, and hence changed the effective pulse duration at the sample.

Figure 3B. Fluorescence yield from PCF-enhanced and conventional Ti:Sapphire two-photon excitation of guinea pig small intestine muscularis externa labelled with anti-smooth muscle myosin and Alexa 594. A 7.3-times increase in fluorescence signal intensity was measured.

Figure 3C. By attenuating the PCF-assisted source, a near-quadratic relationship (gradient of $1.8 \pm 0.1, n=20$ ) between average fluorescence signal intensity and increasing average laser power was observed while imaging guinea pig small intestine muscularis externa labelled with anti-smooth muscle myosin and Alexa 594. This confirmed the nonlinear nature of the excitation mechanism.

Figure 4A. Smooth muscle cells in intact, living rat pulmonary artery, labelled with the voltage sensitive dye di-8-ANEPPS were imaged using TPLSM. With an average power of $22 \mathrm{~mW}$ from both systems directed into the scanhead, (a) shows the image obtained with the PCF-enabled source at a grating spacing of $14 \mathrm{~mm}$ while (b) depicts the image generated using the conventional Ti:Sapphire laser (b).

Figure 4B. Fluorescence yield from PCF-enhanced and conventional Ti:Sapphire TPLSM of intact, living rat pulmonary artery, labelled with the voltage sensitive dye di-8-ANEPPS. A 7.8times increase in fluorescence signal intensity was routinely observed. 


\section{Figures}

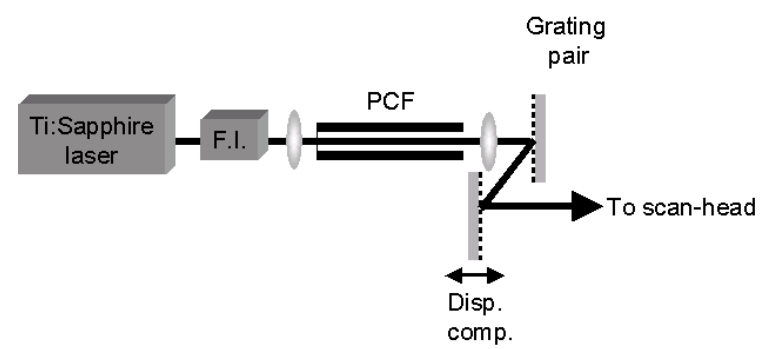

Figure $1 \mathrm{~A}$.

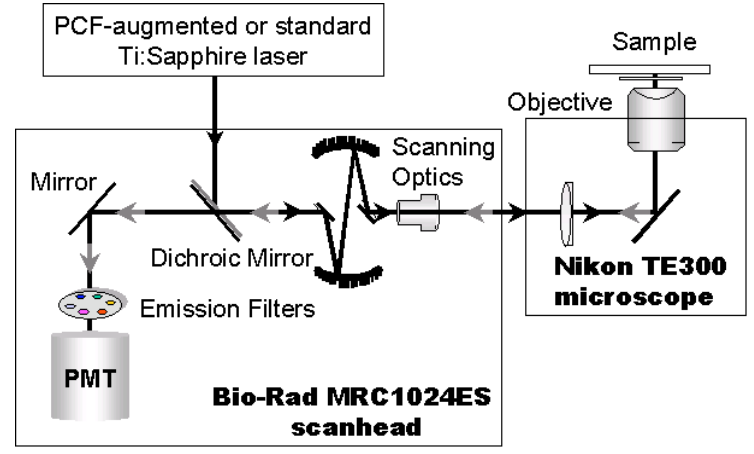

Figure 1B. 


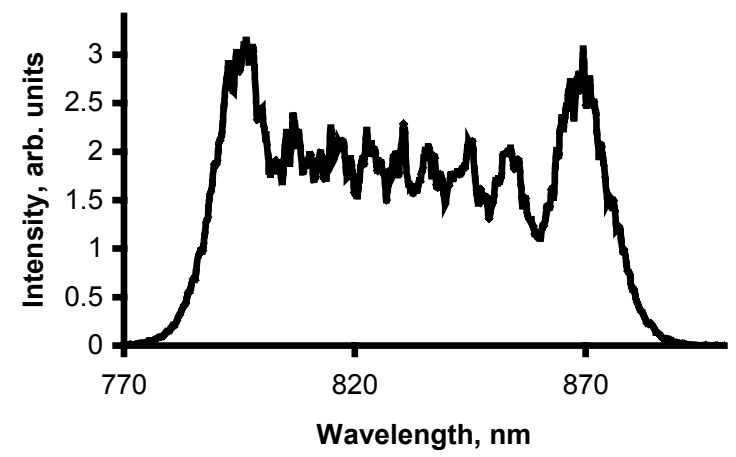

Figure 2A.

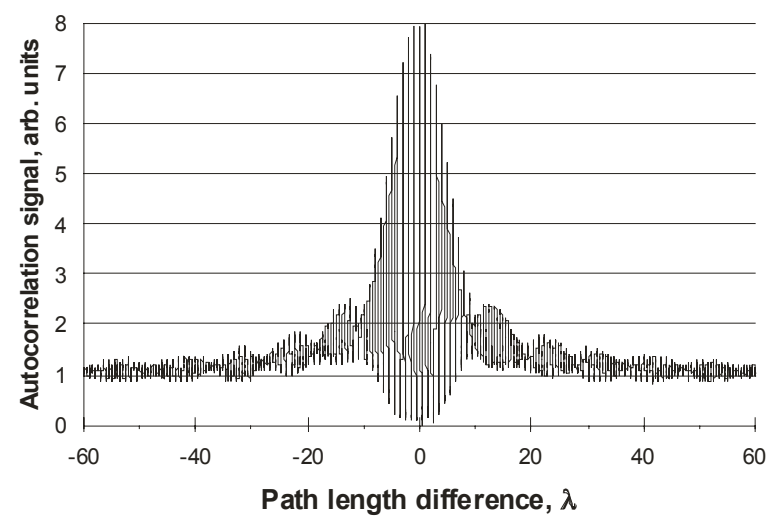

Figure 2B. 

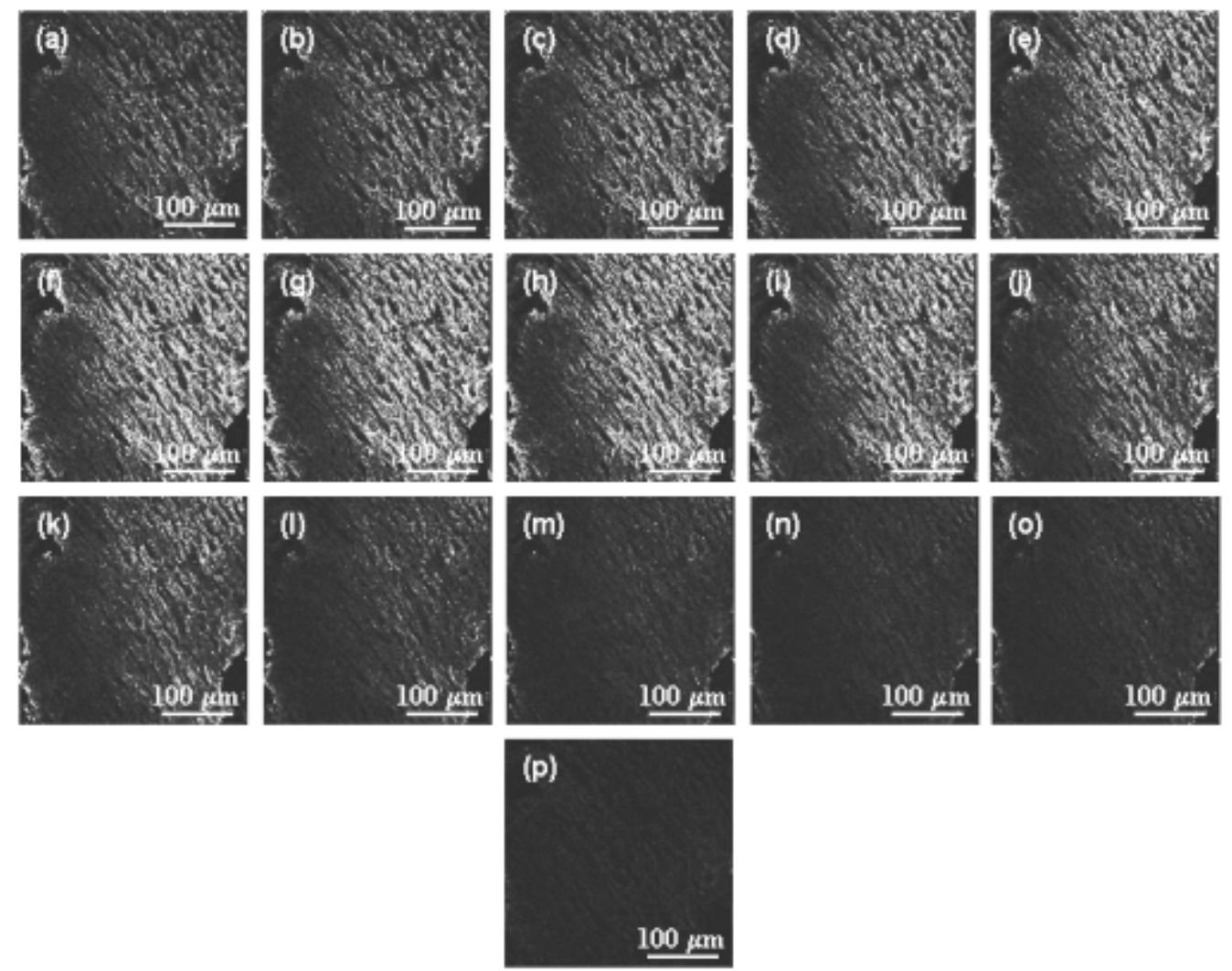

Figure 3A.

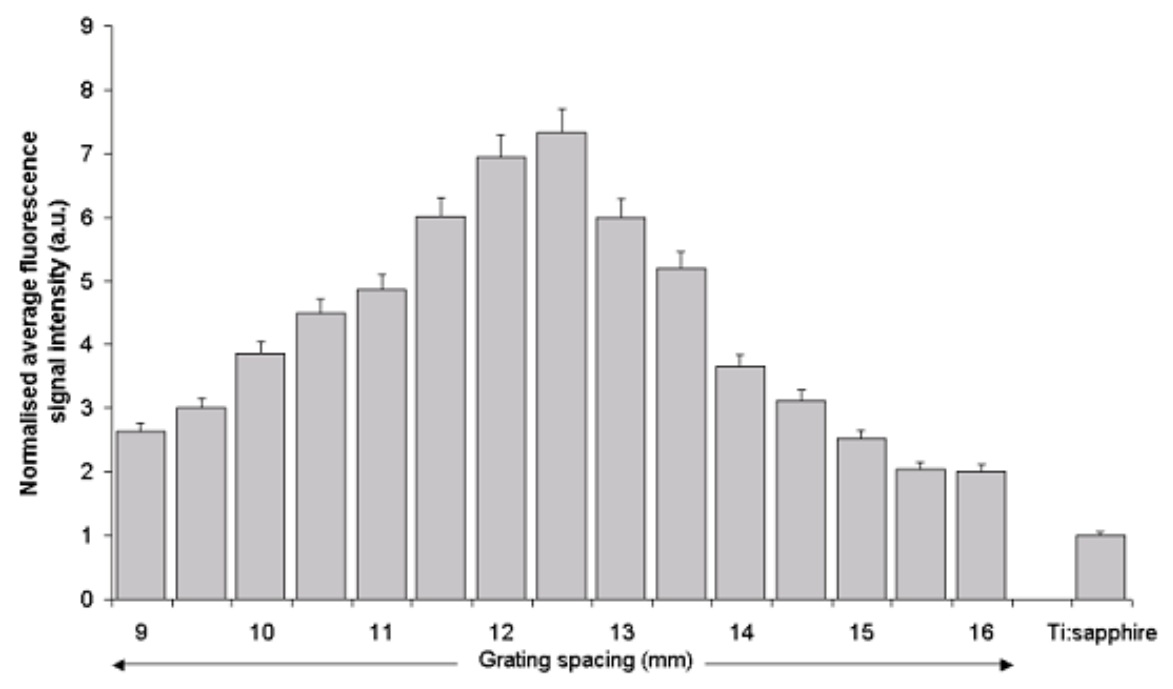

Figure 3B. 


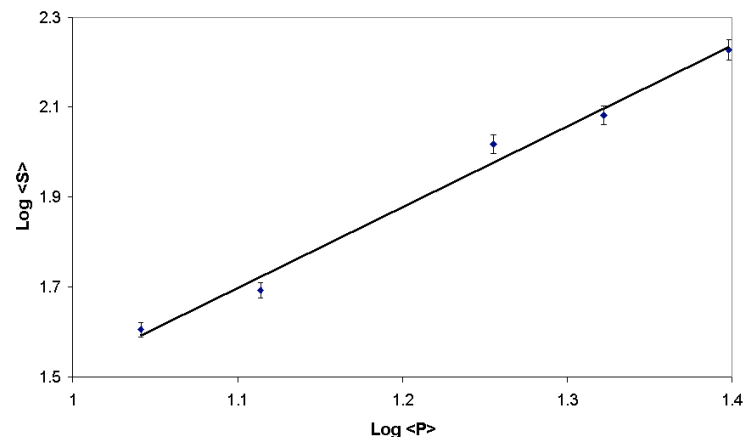

Figure 3C.
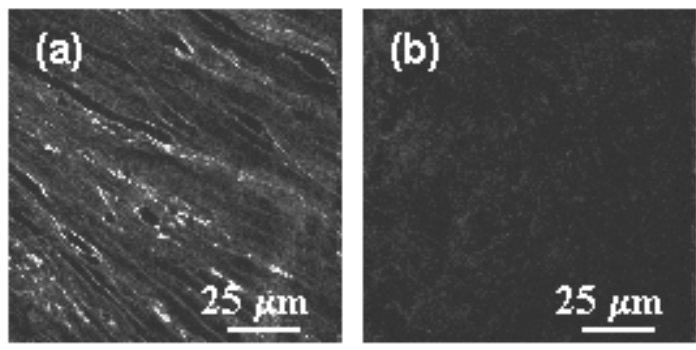

Figure 4A.

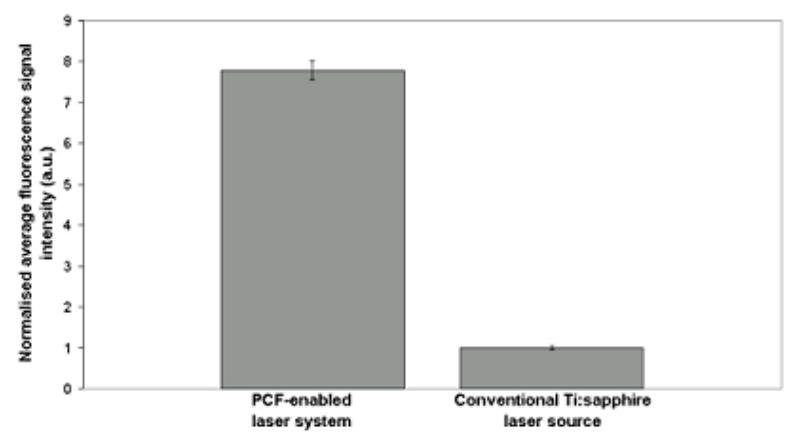

Figure 4B. 